\title{
Effects of Listening of "Dengaku" Music (One of Japanese Traditional Music) to the Changes of Heart Rate Variability
}

\author{
Satoshi Watanabe ${ }^{\mathrm{a}, *}$, Takahiro Sugiyama $^{\mathrm{b}}$, Naofumi Nakaya $^{\mathrm{c}}$ and Naruki Shirahama ${ }^{\mathrm{d}}$ \\ ${ }^{a}$ School of Health Sciences at Odawara, International University of Health and Welfare \\ 1-2-25, Shiroyama, Odawara-shi, Kanagawa, 250-8588, Japan \\ ${ }^{\mathrm{b}}$ Graduate School of Science and Technology, Shizuoka University \\ 3-5-1, Johoku, Naka-ku, Hamamatsu-shi, Shizuoka, 432-8011, Japan \\ 'Faculty of Health Sciences,Tsukuba International University, 6-20-1, Manabe, Tsuchiura-shi, Ibaraki, 300-0051, Japan \\ dDepartment of Creative Engineering, National Institute of Technology (KOSEN), Kitakyushu College \\ 5-20-1, Shii, Kokuraminami-ku, Kitakyushu-shi, Fukuoka, 802-0985, Japan \\ *Corresponding Author: satoshi-w@iuhw.ac.jp
}

\begin{abstract}
To investigate the effects of listening to music, analyses of heart rate variability (HRV) by discrete Fourier transform (DFT) have been widely conducted. These methods are useful to estimate the autonomic nervous system activity (sympathetic or parasympathetic activity). And to examine the effects of listening to "Dengaku" music, one experiment is carried out. Eleven healthy Japanese people participate in the experiment, and one piece of "Dengaku" music is employed as the test piece. All participants are asked to listen to the test piece individually, and their HRV are recorded and analyzed by DFT. The experiment result shows that the sympathetic nerve activities of all participants tend to decrease when they are listening to the test piece. This fact is a rare case (usually, there are some participants whose sympathetic nerve activities tend to increase when they are listening to music). And unique observation (i.e., all LF/HF indexes are decreased), and common observation (i.e., variation of LF/HF indexes are reduced) are obtained when participants are listening to "Dengaku" music. However, to obtain reliable conclusions, conducting more investigations would be especially important.
\end{abstract}

Keywords: "Dengaku" music, heart rate variability, sympathetic nerve activity.

\section{Introduction}

The effects of music listening to improve quality of life (QOL) for heart rate variability (HRV) have been, gradually, confirmed and accepted by many people ${ }^{(1)}$. However, factors of the effects of listening to music on HRV are not clear. To solve this problem, investigations about listening to various music are important. Furthermore, because western music is the most familiar music in daily life, this music is mainly used in previous studies.

On the other hand, for example in Japan, we can recognize a lot of Japanese traditional music (and these styles are not the same as western music), "Dengaku" music, "Min-yo" music, "Ohayashi" music, "Gagaku" music, and so on. Therefore, investigations about the effects of listening to Japanese traditional music are also important.

From these points, the author focuses on "Dengaku" music as one of Japanese traditional music, and the effects of listening to "Dengaku" music are investigated by analyses of $\mathrm{HRV}^{(1)}$.

The remainder of this paper is organized as follows. Section 2 describes "Dengaku" music. The theory of HRV analysis is shown in Section 3. Experiments are given in Section 4. Section 5 shows results and discussion, and this paper reaches the conclusions and future works in Section 6. 


\section{About "Dengaku" Music (2)}

A "Dengaku" music is one of Japanese traditional music. This music is widely distributed in Japan, so there is a lot of "Dengaku" music in the area. The main purposes of using this music are praying to a local God, wishing for a good harvest, and praying for the happiness of the residents. Therefore, the existence of "Dengaku" music is well known to Japanese people.

For recording and inheriting the culture and development of tourism resources, the author is investigating "Dengaku" music, especially "Nishi-ure Dengaku" music in Shizuoka prefecture ${ }^{(3)}$. The "Nishi-ure Dengaku" music (see Fig.1) has more than 1300 years of history, and this music was designated as the 1st important intangible cultural property in Japan in 1976.

Thus, as a first attempt at the effects of listening to "Dengaku" music on the changes of HRV, the author chose "Nishi-ure Dengaku" music as the music for use in this study.

\section{Theory of HRV Analysis ${ }^{(1)}$}

It can be regarded as synonymous with the pulse wave and heart rate analysis in healthy participants ${ }^{(4)}$. The pulse wave can be measured easier than the heart rate. Therefore, it is possible that HRV analysis is carried out by pulse wave analysis. In this paper, the pulse wave analysis is employed as an analysis method instead of HRV analysis.

The peak of the pulse wave is obtained from the photoelectric pulse wave sensor attached to an earlobe or a finger of the subject. The pulse wave intervals (PI) are obtained from the peak of the pulse wave (see Fig.2).

To proceed with a pulse wave analysis from the obtained PI, it is necessary to convert them to time series data (that is, the obtained PI will be interpolated to time series data), because they are not a state of complete time

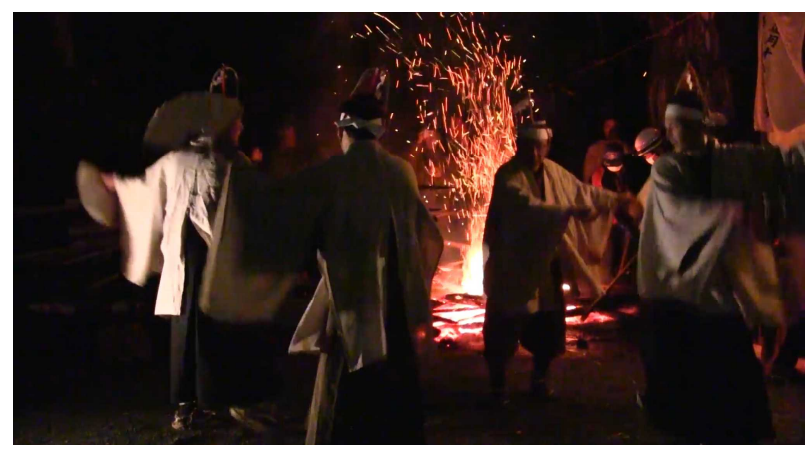

Fig. 1. "Nishi-ure Dengaku" series (not the isochronous intervals). After the conversion step, the PI data in the time series is analyzed by the DFT. At the first step, the frequency spectrum is obtained by DFT as shown in Eq. (3), the following components are extracted from them ${ }^{(5)}$.

LF (Low Frequency) components: $0.04 \mathrm{~Hz}-0.15 \mathrm{~Hz}$

HF (High Frequency) components: $0.15 \mathrm{~Hz}-0.40 \mathrm{~Hz}$

Subsequently, the periodogram is applied to estimate the power spectral density (PSD) of these regions (see Eq. (4) and (5))

$$
\begin{aligned}
& X[k]=\sum_{n=0}^{N-1} x[n] e^{-j \frac{2 \pi k n}{N}},(k=0,1,2, \cdots, N-1) \\
& L F=\frac{\sum\left|X_{L F}\right|^{2}}{f_{s} N}, \quad X_{L F}=\left\{X[k] \mid 0.04 \mathrm{~Hz} \leq \frac{k f_{s}}{N} \leq 0.15 \mathrm{~Hz}\right\} \\
& H F=\frac{\sum\left|X_{H F}\right|^{2}}{f_{s} N}, \quad X_{H F}=\left\{X[k] \mid 0.15 \mathrm{~Hz} \leq \frac{k f_{s}}{N} \leq 0.40 \mathrm{~Hz}\right\}
\end{aligned}
$$

From the calculation result of Eq. (4) and (5), autonomic nervous activity (sympathetic or parasympathetic activity) indexes can be estimated as follows $^{(5)}$.

$$
\begin{gathered}
\text { Sympathetic activity index }=\mathrm{LF} / \mathrm{HF} \\
\text { (the ratio of (4) to (5)) }
\end{gathered}
$$

Parasympathetic activity index $=\mathrm{HF}$ (the value of (5))

That is, the autonomic nervous activity is estimated by the value of Eq. (4) and (5). According to the above preparation, the autonomic nervous activity by listening to "Dengaku" music can be estimated.

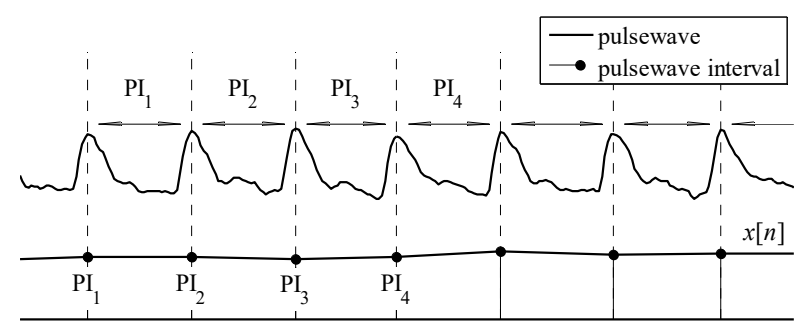

Fig. 2. Pulse wave and Pulse wave interval 


\section{Experiments $^{(1)}$}

To examine the effects of listening to "Dengaku" music on the changes of HRV, one experiment is carried out. Eleven healthy people participated, and one piece of

"Dengaku" music ("Detai-Doji" music) is employed as the test piece. All participants are asked to listen to the test piece individually, and their HRV are recorded and analyzed by DFT.

\subsection{Participants}

Eleven healthy Japanese people (age: $26.27 \pm 10.97$ (average \pm standard deviation), nine males and two females) are invited as participants.

Ethical considerations are as follows:

The explanation of this study was given by the oral and documents to the participants, and it was also given that participation in this study was voluntary. Furthermore, the data have been collected anonymously, and have been only used carefully for the presentation of this study.

\subsection{Experiment Environment}

The experiment is conducted in the daily living environment such as office, classroom, and living room. The experiment employs the headphone (earphone) and a photoelectric pulse wave sensor attached to a finger of the participants. The participants are requested to sit and do the experiment in closed-eye condition. No other special instruments are employed.

\subsection{Test Piece ("Dengaku” Music)}

The author chose the "Detai-Doji" music as the test piece for use in this study from the "Nishi-ure Dengaku" music. The "Detai-Doji" music has been obtained from the "Nishi-ure Dengaku" live recording. And its fluctuation is examined as follows. First, the test piece is loaded into the personal computer (OS: Windows) for analysis. Next, its instantaneous frequencies are obtained by the zero-crossing method. Then power spectrum intensities $P$ are determined using them. Finally, logarithmic plots of $P$ vs. frequencies $f$ are expressed. These analyzes are conducted by the software tool - "Yuragi-Analyzer" ${ }^{(6)}$. The results are summarized in Fig. 3 and 4.

The slope value, $\lambda$ is determined from Fig. 4 . It is found that the $\lambda$ value for the test piece is 0.429 . That is, it can be considered that the test piece is not $1 / f$ fluctuation music.

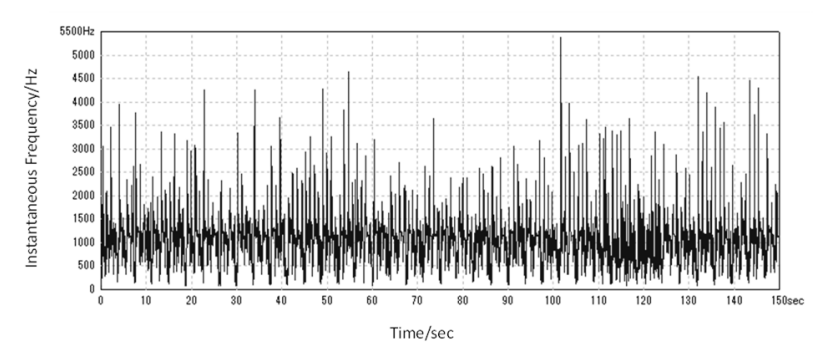

Fig. 3. Instantaneous Frequencies of Test Piece

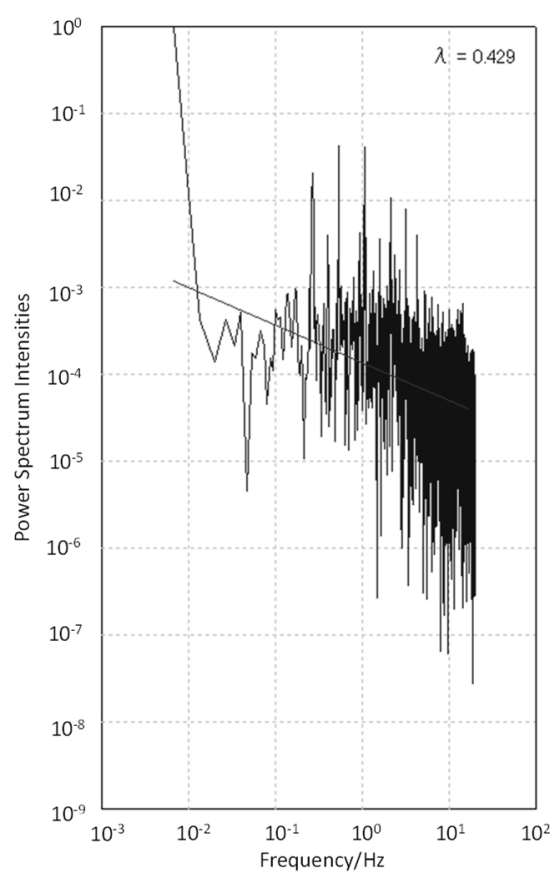

Fig. 4. Logarithmic Plots of $P$ vs. $f$

\subsection{Procedures of the Experiment}

The "Detai-Doji" music is employed as the test piece. To play the test piece, a personal computer (OS: Windows) is employed. And 11 participants (see 4.1) are asked to listen to the test piece individually.

The flow of the experiment is shown in Fig. 5. The peak of the pulse wave obtained from the photoelectric pulse wave sensor (U-MEDICA PDU-M100) is attached to the finger of the participants. Then, their HRV are recorded and analyzed by software (U-MEDICA “ARTETT").

\section{Results and Discussion}

All participants' LF/HF indexes (see Section 3) have been obtained from the experiment. They are divided into two stages: before (before listening to the test piece), and 


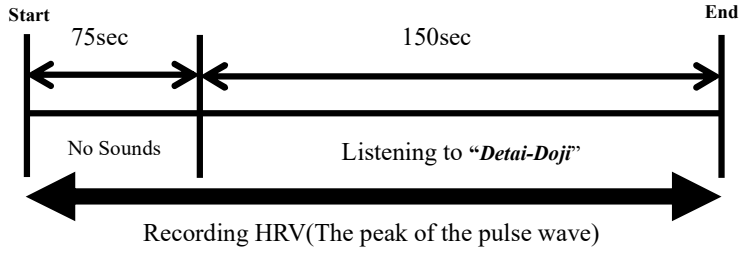

Fig. 5. The Flow of the Experiment

stimulation (listening to the test piece). Fig. 6 shows the results of this process, and these values are also summarized in Table 1. When the participants are listening test piece, it is shown that all LF/HF indexes (the indexes of sympathetic nerve activities) are decreased. This fact is a rare case $^{(1)}$. Usually, there are some participants whose $\mathrm{LF} / \mathrm{HF}$ indexes tend to increase when they are listening to music, the ratio of them is about $30 \%$ of all participants ${ }^{(1)(7)}$.

By the way, the decrements of these indexes are also summarized in Table 1. It can be seen that these values are different from participants, some trends do not exist in Table 1.

Here, let us consider comparing this experimental result and the author's previous study that result is as same as this study ${ }^{(1)}$. These elements are summarized in Table 2 . From Table 2, it is found that the common element of both is repeating a certain rhythm. So, it is suggested that this common element may lead to the experimental result (that is, all $\mathrm{LF} / \mathrm{HF}$ indexes are decreased).

On the other hand, it also seems that variation of $\mathrm{LF} / \mathrm{HF}$ indexes is reduced when the participants are listening test piece in Fig. 6 (also shown in Table 1). This observation can be seen in previous studies usually(1)(5)(7).

From these facts, it can say that sympathetic activity is decreased significantly when the participants are listening to "Detai-Doji" music ( "Dengaku" music). More, there are two types of suggestions from these facts, one is unique

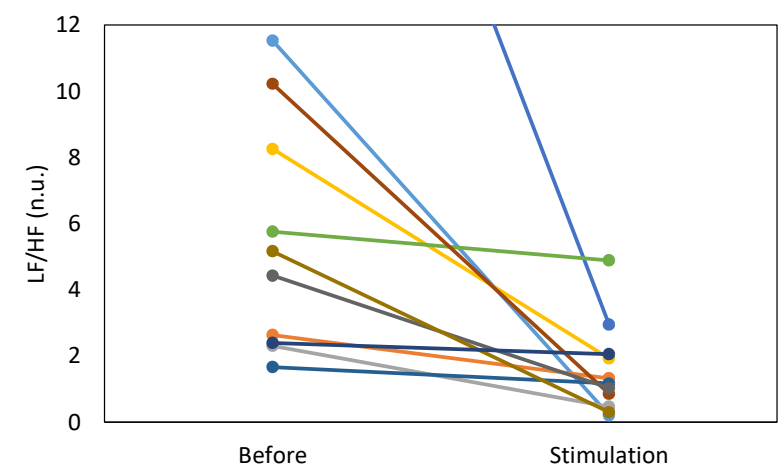

Fig. 6. Changes of LF/HF Indexes in the Experiment
Table1. Changes of LF/HF Values in the Experiment

\begin{tabular}{|c|r|r|r|}
\hline ID & Before & Stimulation & $\begin{array}{c}\text { Decrements } \\
\text { (From Before to } \\
\text { Stimulation) }\end{array}$ \\
\hline 1 & 11.531 & 0.208 & 0.982 \\
\hline 2 & 2.637 & 1.326 & 0.497 \\
\hline 3 & 2.302 & 0.470 & 0.796 \\
\hline 4 & 8.257 & 1.926 & 0.767 \\
\hline 5 & 29.466 & 2.950 & 0.900 \\
\hline 6 & 5.753 & 4.890 & 0.150 \\
\hline 7 & 1.669 & 1.167 & 0.301 \\
\hline 8 & 10.222 & 0.856 & 0.916 \\
\hline 9 & 4.430 & 1.027 & 0.768 \\
\hline 10 & 5.170 & 0.305 & 0.940 \\
\hline 11 & 2.392 & 2.055 & 0.141 \\
\hline \hline Max. & 29.466 & 4.890 & 0.982 \\
\hline Min. & 1.669 & 0.208 & 0.141 \\
\hline Median & 5.170 & 1.167 & 0.768 \\
\hline Standard & 7.599 & 1.314 & 0.306 \\
Deviation & & & \\
\hline & & & \\
\hline
\end{tabular}

Table2. Comparing This Study and the Previous

\begin{tabular}{|c|c|c|}
\multicolumn{3}{c}{ Study $^{(1)}}$. \\
\hline Music & $\begin{array}{c}\text { This Study } \\
\text { "Dengaku" } \\
\text { Music }\end{array}$ & $\begin{array}{c}\text { Mrevious Study } \\
\text { Band Music) }\end{array}$ \\
\hline $\begin{array}{c}\text { l/f fluctuation } \\
\text { Repeating a } \\
\text { Certain Rhythm }\end{array}$ & Yos & Yes \\
\hline $\begin{array}{c}\text { Listening at the } \\
\text { same time }\end{array}$ & No & Yes \\
\hline
\end{tabular}

observation (i.e., all LF/HF indexes are decreased), another is a common one (i.e., variation of $\mathrm{LF} / \mathrm{HF}$ indexes are reduced). However, it is necessary to consider whether this effect is caused by listening to the test piece or not. In addition, the participants are all Japanese people, it is necessary to extend the participants of this study to not only Japanese but also foreigners. That is, more studies in the future are important.

\section{Conclusions}

To examine the effects of listening to "Dengaku" music, one experiment is carried out. The experiment result shows that the sympathetic nerve activities of all participants decreased when they are listening to "Dengaku" 
music.

From this result, this study concludes two suggestions: (1) listening to "Dengaku" music has a significant effect of decreasing sympathetic nervous activity; (2) unique observation (i.e., all $\mathrm{LF} / \mathrm{HF}$ indexes are decreased), and common observation (i.e., variation of $\mathrm{LF} / \mathrm{HF}$ indexes are reduced) are obtained when participants are listening to "Dengaku" music. However, it is necessary to consider whether these two suggestions are caused by the conditions of the above experiment or not. And to obtain reliable conclusions, applying this method in future studies and conducting more investigations are especially important.

Future works are as follows: (1) it is necessary to extend the participants of this study to not only Japanese but also foreigners. (2) the essence of the effects of listening to music, (3) variation of Japanese traditional music.

\section{References}

(1) Satoshi Watanabe, Ryouichi Takaue, Fenghui Yao, Yuji Matsumoto, Hiroyuki Tsukamoto, Naruki Shirahama, Naofumi Nakaya and Yukio Mori: "Effects of 1/f Fluctuation Music Listening on Autonomic Nervous System Activity", Journal of the Institute of Industrial Applications Engineers, Vol.6 No.2, pp.86-91, 2018

(2) Yuko Yoshikawa: "Nishi-ure Dengaku no Minzoku Bunka-ron", 270 pages, Iwata-Shoin (Tokyo), 2012 (in Japanese)

(3) Anri Hikosaka, Satoshi Watanabe and Takahiro Sugiyama: "Analysis of Impressions about Intrinsic Information and Its Order of Presentation to Improve Motivation to Visit "Nishiure-Dengaku"', Proceedings of the 20th Research Meeting of the Society for Tourism Informatics, pp. 56-59, 2019 (in Japanese)

(4) Toshiki Kikuchi, Yuji Sano, Jyunichi Abo, Shinsuke Urushidani and Tomoe Yamazaki: "Evaluation of Autonomic Nervous Activity using the Second Derivative Photoplothysmograph a-a Interval", Journal of Sport Sciences and Osteopathic Therapy, Vol. 10, No. 3, pp.177-183, 2009 (in Japanese)

(5) Kiyokazu Hori, Yasutoshi Senga, Satoshi Minami and Seiki Hori: "Effects of listening to music on heart rate variability” , Japanese Journal of Biometeorology, Vol. 41, No. 4, pp.131-140, 2004

(6) "Yuragi-Analyzer":

http://mahoroba.logical-arts.jp/category/software/yurag i-analyzer, as of 1 July 2021 (in Japanese).
(7) Satoshi Watanabe, Yuji Matsumoto, Masashi Tomita and Yukio Mori: Heart Rate Variability Analysis and the Subjective Evaluation by Visual Analog Scale for Subjects Listening 1/f Fluctuation Music, Journal of Biomedical Fuzzy Systems Association, Vol. 15, No. 2, pp.1-10, 2014 (in Japanese). 